\title{
CONDOMINIOS Y ESTABILIDAD ECOLÓGICA EN LOS ANDES CENTRALES COLOMBIANOS
}

\author{
Juan Leonardo González Plazas ${ }^{1}$ \\ Universidad de Antioquia, Instituto de Estudios Regionales INER \\ Grupo de Estudios del Territorio \\ juangonzalez@iner.udea.edu.co
}

Manizales, 2009-10-27 (Rev. 2009-11-06)

\begin{abstract}
RESUMEN
El siguiente artículo expone una caracterización socio-espacial sobre la propagación espacial de los proyectos residenciales, denominados condominios, su papel en las alteraciones de las capacidades ecológicas para el abastecimiento hídrico y la transferencia de biodiversidad entre los ecosistemas localizados en los andes centrales colombianos. De forma comparativa, se estudia la tendencia de dispersión urbana de los sistemas urbanos del eje cafetero (Manizales, área metropolitana de Pereira, Armenia) así como la tendencia de expansión urbana entre el área metropolitana del Valle de Aburrá y el altiplano del oriente antioqueño. Como resultados principales, se destaca la pérdida de la capacidad espacial de los fragmentos de ecosistemas para conectarse, debido a la localización de procesos urbanísticos e infraestructurales que se interponen, dificultades de los ecosistemas en su regulación hídrica, afectación de las capacidades hidrogeológicas tras la ausencia de planes de alcantarillado, y la ausencia de seguimiento y regulación por parte de la planeación urbana sobre los anteriores aspectos.
\end{abstract}

\section{PALABRAS CLAVES}

Dispersión urbana, condominios, corredores ecológicos, ecosistemas andinos.

\section{CONDOMINIUMS AND ECOLOGICAL STABILITY IN THE CENTRAL COLOMBIAN ANDES}

\begin{abstract}
The following article presents a socio-spatial characterization of the spatial spread propagation of the socalled residential condominiums and itstheir role in the alterations of ecological capacities for water supply and biodiversity transfer of biodiversity between ecosystems located in the central Andes in Colombia. By means of comparative studies, the trend of urban sprawl trend of urban systems in the ecocCoffee Growing Areatown (Manizales, Pereira metropolitan area, Armenia) and the trend of urban expansion trend between the metropolitan area of the AburraBore Valley and the highlands of eastern Antioquia. As mMain results include highlighted the loss of spatial ability ofto connect ecosystems fragments of ecosystems to connect, due to the location of urban processes and infrastructure processes that arisestand, such as the difficulties of ecosystems in water regulation, involvement of hydrogeological skills behind after the absence of plans sewage plans, and the lack of monitoring and regulation of urban planning on the aboveregarding these aspects.
\end{abstract}

KEY WORDS: Urban sprawl, condominiums, ecological corridors, Andean ecosystems.

\section{INTRODUCCIÓN}

La dispersión urbana es una realidad dada en sistemas urbanos de países desarrollados y en vía de desarrollo, claro está, ambos diferentes en las formas, aplicaciones y continuidad de la planeación sistemática de la expansión urbana (control derrame de plusvalías, proyección físico-espacial de la expansión, coherencia con los usos del suelo, prevención, corrección, mitigación, compensación en materia ambiental). En los dos sentidos, la dispersión urbana ha llevado a un debilitamiento de las ruralidades que gravitan en torno a estos sistemas, especialmente en las ciudades latinoamericanas. La debilidad institucional en torno a la planeación y, además, la imposición de modelos de desarrollo económicos ligados a la globalización, son circunstancias que han presionado por un cambio en las lógicas de ocupación del espacio y del consumo de los recursos naturales. 
En la última década, sistemas urbanos como el área metropolitana de Aburrá y los sistemas urbanos intermedios del eje cafetero (Manizales, Pereira y Armenia) han experimentado ampliaciones considerables de sus áreas de influencia, que son tangibles por la transformación de los usos del suelo (de rural a urbano), y por el debilitamiento de las conexiones ecológicas locales dadas especialmente en sus diferentes vías de acceso $^{2}$.

Esta dispersión plantea un desencuentro entre lo que los mundos rural y urbano perciben sobre el valor de los recursos (agua, suelo, ecosistemas) frente a la oferta y la demanda. El desarrollo espacial urbano tiende a subestimar tanto la permanencia, como las conexiones espaciales entre ecosistemas estratégicos que, sin lugar a dudas, son de vital importancia en el tema del abastecimiento hídrico localregional por medio de la redefinición del uso del suelo, pasando de ser un recurso ecológico destinado principalmente al cultivo, a un uso estrictamente residencial, industrial o de servicios.

Sobre lo anterior, surge una pregunta: ¿De qué manera la dispersión urbana, y en especial la propagación dispersa de los condominios, irrumpe en la estabilidad ecológica local y regional? La conjunción de las dinámicas referidas, y que en la actualidad son visibles en la composición del paisaje cultural, advierten para un futuro no lejano una proliferación de actividades urbanas dispersas, preferiblemente de orden residencial, de estratos socioeconómicos solventes y que, a manera de encerramientos, establecen distancias y formas de segregación socio-espacial frente a los reductos de ruralidades establecidas en varios lugares.

\section{METODOLOGÍA}

El presente trabajo hace parte de una propuesta que desde la Biogeografía y la Geografía urbana se interesa en identificar dinámicas del deterioro ecológico por parte de tendencias dispersas de vivienda periurbana, inicialmente en el eje cafetero y en Antioquia. Por medio de varios resultados de campo, realizados tanto en el ejercicio de docencia en los programas de Geografía urbana y Biogeografía de la Universidad de Caldas, en la participación de distintas actividades en los comités técnicos de la ecoregión eje cafetero ${ }^{3}$, como en la participación de las agendas técnicas sobre planeación urbano regional en Medellín y el oriente antioqueño ${ }^{4}$-entre los años 2003 y 2009-, se ha llegado a compilar información que ha permitido inicialmente hacer un bosquejo comparativo acerca del estado de la espacialidad de la dispersión urbana en varios contextos.

Los resultados establecen inicialmente la formulación de un modelo de dispersión para cada área de estudio, construido sobre las características geomorfológicas, la identificación de las cuencas hidrográficas comprometidas, la red de conexión vial, la espacialidad de los remanentes andinos y la identificación de los municipios que participan en esta dinámica; aspectos que terminan graficándose como coremas o modelos gráficos de organización espacial.

Estos modelos representan el uso del suelo actual de las áreas de estudio, generalizada en escala 1:400.000 y que utilizan la nomenclatura del modelo de coberturas terrestres CORINE LAND COVER. Se relacionan también los resultados del análisis sobre la composición ecológica y social de las unidades de paisaje involucradas en la dispersión urbana y la identificación de subcentros articulados por las redes de accesibilidad.

Igualmente se exploran las falencias de los planes de ordenamiento territorial en cuanto a la reglamentación y normativas referentes al manejo de las áreas de expansión, al igual que las interpretaciones políticas en torno al desarrollo subregional en las áreas de estudio.

\section{DISPERSIÓN URBANA Y CONDOMINIOS}

Actualmente se establece un nuevo modelo de ciudad definido por DEMATTEIS (1998:17) como la ciudad difusa, que lleva a una redefinición de la cuestión urbana. Según HARVEY (1985: 53) se trata de “... procesos sociales que producen y reproducen espacio temporalidades especificas y muy heterogéneas que son a menudo de tipo radicalmente nuevo y distinto en sus tipos de acción social....". 
La periferia está definida físicamente por la materialización espacial de los contrastes ambientales y empalmes de modos de vida entre el hábitat urbano y el rural. Para GÓMEZ (1995: 46) el paisaje periurbano es una heterogeneidad de usos con ruralismo residual, tanto de actividades mixtas rurales como urbanas. El contraste espacial resalta diferenciaciones socio-económicas que diseñan diferentes entornos o periferias encajadas unas en otras, formando una jerarquía espacial de sub paisajes al interior del paisaje cultural urbano.

BARROS (1998:10) ha formulado dos grandes contextos que conforman la interface urbano rural. Un primer contexto influenciado directamente por la proximidad del núcleo urbano principal, donde es evidente la expansión acelerada de la red urbana, y un segundo (el cual se quiere desarrollar en esta ocasión), caracterizado por la permanencia de ruralidades que en la actualidad experimentan la inserción de diversos elementos y actividades urbanas y que cumple un papel fundamental en el abastecimiento energético (alimentos, materia prima) y que ella denomina cuencas de abastecimiento.

En los últimos 20 años, la producción de estudios periurbanos y su relación con el medio ambiente ha hecho hincapié en el tema del riesgo físico de las inundaciones y los deslizamientos. En otros casos, varios estudios profundizan en el tema del hábitat en la periferia, orientados hacia la familia, las relaciones comunitarias, la violencia, los problemas por el acceso al agua, la condición alimentaria, los asentamientos precarios y la degradación de los ecosistemas.

A nivel global resalta la producción de ALLEN (2003), DE MATTOS (2001), DAVILA (2003) sobre los impactos ambientales-sociales y las políticas que inciden en el desarrollo de las interfaces urbano rurales; también TACOLI (1998) acerca de los vínculos económicos urbanos y rurales en la periferia. El trabajo de BETTINI (1998), "Ecología urbana", compila una serie de tendencias de estudio experimental en hábitat y sostenibilidad ecológica por medio del uso del suelo para conservación de bosques en los bordes urbanos, a partir de elementos de análisis sistémico-metabólico, considerando el sistema urbano como organismo.

Para el caso local-regional sobresalen los trabajos de HERMELIN y RENDON (2007) sobre las relaciones entre expansión urbana y aspectos geomorfológicos que permiten o restringen el fenómeno, analizando a la vez las alteraciones al medio físico y problemas asociados al riesgo, no sólo para el caso del área metropolitana del valle de Aburra y sus dispersiones, sino también para otros sistemas urbanos en Colombia.

También se refiere el trabajo apoyado por la cámara de comercio del oriente antioqueño a raíz de las preocupaciones de diferentes gremios económicos de la subregión y en el cual también figura HERMELIN (2008). Este trabajo trata sobre las realidades y perspectivas del valle de San Nicolás, expone una serie de dinámicas tanto ambientales como sociales, dadas por la dispersión urbana y las vías posibles de planificación urbano-regional de estos aspectos.

Por último, se cita el trabajo sobre indicadores para medición de huellas ecológicas y sostenibilidad urbana de AGUDELO (2005) donde el autor, por medio de un estudio urbano territorial, analiza el crecimiento urbano del valle de Aburrá y las capacidades ambientales de su entorno, aspecto que aporta para la comprensión de dinámicas ecológicas urbanas externas y que inciden en aspectos ambientales y urbanos del altiplano de Rionegro.

Para el caso de estudio, el proceso de la peri-urbanización en la última década ha sido evidente. El influjo de las economías globales en cada una de las ciudades del eje cafetero ha derivado visiones y modelos de desarrollo local y regional sobre los modos de producción y las tendencias de consumo de mercados. Y es de esta forma como las estructuras agrarias que circundaban las ciudades comenzaron a debilitarse en sus formas de tenencia de la tierra, del trabajo y de sus vínculos con la naturaleza.

La inserción del efecto globalizador en la primera década del siglo XXI, ha mostrado visibles rasgos espaciales de su rápida inserción en el mundo rural y que, con propósitos de mejoramiento de la calidad de vida, ha reconfigurado el modo de producción, las formas de usos del espacio y sus recursos, por medio de la extensión de actividades y elementos urbanos propios de la ciudad en el campo. Las ampliaciones infraestructurales para la movilidad, el acceso más inmediato a créditos para diversos consumos y las nuevas tecnologías de comunicación, entre otros, han sido factores clave para entender la lógica del posicionamiento de mercados y estilos de vida urbana en las áreas de estudio. 
Es de esta forma como la elección de vivienda por parte de clases sociales económicamente solventes, está determinada por los factores anteriormente descritos agregando, además, las posibilidades económicas que permiten decidir dónde se desea habitar con respecto a la ciudad, el grado de aislamiento que se quiere obtener en relación con ciertos problemas urbanos directos (contaminación, delincuencia, densificación, entre otros) y el poder de elección sobre cuánto espacio se quiere consumir por parte de una familia o individuo para desarrollar ampliamente dentro de su morada sus espacios sociales e íntimos.

El trabajo define al condominio como un modelo urbano compacto, un programa de vivienda de encerramiento con un diseño orgánico ${ }^{5}$, distanciado del casco urbano consolidado, pero que está articulado por un vía principal o secundaria. El condominio son unidades residenciales donde las relaciones de comunidad son austeras, si se compara con la construcción del colectivo e identidad de un "barrio" y que, tal como lo plantea LEFEBVRE (1979:181), tiene una tendencia: "a la ausencia de una vida social espontánea y orgánica, que presiona a una privatización absoluta de la existencia".

Tal vez la tendencia post-urbana conduce a nuevas formas de relación con los vecinos y los entornos, en el momento tímidamente estudiadas por las ciencias humanas y el urbanismo en el país, y en las que las asociaciones de vecindad sólo se limitan a cuestiones administrativas del encerramiento, hecho que incide sobre la percepción de las realidades ecológicas y sociales por parte de sus habitantes sobre su entorno.

Generalmente, estos encerramientos son propuestas de vivienda que modifican e intervienen extensiones de suelo entre 10,40 y hasta 400 hectáreas $^{6}$, ofertando parcelaciones con dimensiones que van de los 250, 1000 y 10000 metros cuadrados, dependiendo de las restricciones topográficas de cada sistema urbano, y del costo del metro cuadrado ${ }^{7}$. Esto expone en el conjunto de la organización espacial una tendencia a la brecha social residencial, especialmente en ciudades donde hay escasez de suelo para construir y que, por lo tanto, lleva a un cuestionamiento sobre para cuál población existe tal escasez ${ }^{8}$.

En muchos casos, la localización de los condominios es independiente de los direccionamientos preexistentes en materia de planificación urbana regional, así como de las regulaciones sobre el uso del suelo que dictan los ordenamientos territoriales. Es así como, desde los consejos municipales, se tienden a modificar tanto extensiones como los usos del suelo reglamentados por el POT, con el fin de facilitar los trámites en la solicitud de licencias ambientales y de construcción para los proyectos de vivienda. Igualmente, la localización de éstos tiende a migrar de municipio en municipio cuando se agotan los suelos de uno y otro, o cuando los derrames de valorización para vivienda no son rentables.

\section{CONTEXTO DE ESTUDIO}

El área de estudio corresponde a los escenarios periurbanos dados en los municipios que gravitan alrededor de los tres sistemas urbanos del eje cafetero (Armenia, Pereira, Manizales) y el área metropolitana del valle de Aburrá, hacia un espacio conocido como el altiplano de oriente o valles de San Nicolás.

En total son 22 municipios que, dada su proximidad con el área metropolitana y las ciudades intermedias del eje cafetero, tienden a una acelerada transformación de sus actividades rurales y la localización desagregada de elementos urbanos como los condominios. En términos geomorfológicos, los escenarios de descripción se localizan en las unidades morfogénicas andinas de media montaña (29001.200 m.s.n.m) y baja montaña (1.200-500 m.s.n.m) ${ }^{9}$.

En el oriente antioqueño, el relieve corresponde a un sistema de valles colinados y plano ondulados, con una altitud que varía entre los 2.000 y 2.200 metros de altura. Su condición plano ondulada y su localización estratégica que integra a conexión vial valle del Magdalena-Bogotá, ha permitido una dispersión no planificada en distintos valles de este "altiplano", así como la concentración de zonas francas e industrias en los municipios de Rionegro, Guarne, Marinilla, a lo largo de la autopista que conduce a Bogotá. 
Los municipios integrados comprometidos con esta situación (como el Retiro, Rionegro, Guarne, La Ceja y Marinilla) conforman $846 \mathrm{~km} 2$ de dispersión al interior de la cuenca alta del río Negro. Esta red hídrica es considerada como la estructura principal de drenaje en la región, además de ser el estructurador ecológico del altiplano vertiendo sus aguas al sistema de embalses de Guatapé, localizado al nororiente del altiplano (cuenca regional del río Magdalena)

Los municipios integrados a la dispersión urbana en el eje cafetero son Neira, Villamaría, Manizales, Chinchiná, Santa Rosa, Dosquebradas, La Virginia, Cartago, Alcalá, Quimbaya, Circasia, Filandia, Salento, La Tebaida, Montenegro, Calarcá y, juntos, conforman un área potencial de dispersión 4.892 km2, integrados a la figura de sostenibilidad regional denominada eco-región Eje cafetero.

La eco-región se denomina por la estructura de cuencas hidrográficas que tienen su origen en el complejo glaciar-volcánico de páramos y selvas andinas conocido como el complejo Ruiz Tolima ${ }^{10}$. En este, se inician los sistemas hídricos de los ríos Chinchiná, Otún, Quindío y se integran en formas de relieves de piedemontes y unidades montañosas entre los 5.000 y 900 metros sobre el nivel del mar, en los que han establecido diferentes verticalidades de producción agraria (ganaderías, café, caña, forestal), con remanentes que garantizan los procesos ecológicos entre los páramos y el río Cauca.

A diferencia del altiplano de Rionegro, sus condiciones geomorfológicas de topografías abruptas y suaves, hace que la expansión sea diferencial con problemas de escasez de suelo urbano, densificación poblacional, vulnerabilidad constante por eventos de movimiento en masa, y elevación de costos de construcción.

\section{ESTABILIDAD ECOLÓGICA Y CONDOMINIOS}

En la actualidad, los andes centrales colombianos manifiestan una gran riqueza de biodiversidad a partir del posicionamiento de grandes remanentes ${ }^{11}$ de ecosistemas andinos ${ }^{12}$, además de fragmentos significativos de ecosistemas subandinos ${ }^{13}$ y la presencia de enclaves de ecosistemas secos tropicales $^{14}$ de manera forma muy local, estos últimos, dependiendo de las condiciones de déficit hídrico natural establecidas en el fondo del cañón del río Cauca.

Una situación especial a resaltar radica en que todavía existe la posibilidad de conexión entre cada uno de estos fragmentos, hecho que, en términos de la estabilidad y funcionalidad de los ecosistemas ${ }^{15}$, garantiza la transferencia de valores genéticos y la regulación de la humedad desde los ejes o divisoria de la cordillera hacia el río Cauca. La garantía de la permanencia y conexión, depende principalmente de las disposiciones sobre uso, manejo y conservación de áreas de protección en cada uno de los municipios que administran estos espacios.

Desafortunadamente, al no existir una coherencia en la dirección conjunta entre municipalidades y departamentos sobre este asunto, la cohesión ecológica de estos tiende cada vez a volverse más estrecha, a desaparecer, en especial, en las áreas municipales influenciadas por la dispersión urbana. En esto, la propagación urbana tiene un papel importante en la presión y ruptura de los corredores ecológicos $^{16}$, promoviendo dinámicas de reconversión de usos de suelos rurales para actividades agroindustriales y turísticas, encaminadas sobre propósitos de desarrollo local y regional ${ }^{17}$. Esta variación interviene sobre el funcionamiento de estructuras ecológicas a escalas regionales (1:400.000) en las que se encadenan ecosistemas andinos, sub andinos y bosques secos tropicales que están cohesionados espacialmente por una cuenca hidrográfica.

Como casos concretos, la tendencia de dispersión hacia el oriente en municipios como el Retiro, Rionegro, Guarne y Marinilla en el altiplano (figura 1) se presenta un incremento de inversiones en infraestructura de movilidad, el desarrollo de zonas francas, industrias, sub centros donde convergen pequeños y grandes centros comerciales y una masiva parcelación de predios para el establecimiento de condominios. Individualmente, cada municipio ha definido, desde sus planes de ordenamiento territorial, las formas y los lugares de urbanización, materializándose una red de apolillamiento ${ }^{18}$ en los valles de San Nicolás; sin un desarrollo de áreas de sesión en las vías para efectos de espacio público, áreas de protección para corredores ecológicos y proyecciones de trazados urbanísticos para urbanizar. 


\section{CONVENCIONES}

USOS DEL SUELO: BASE CORINE LAND COVER ESCALA 1:T60.000 - GENERAUZADA
222 Cana de azvar
22.4 Pistano y Banso
22.5 Colle
24.3 Mosaco de pastos, cubves y espacos naturales
23.2 Pastos arbolados
21 Zonas ndustriales 0 comerciles
24.1 Mosaico de catives
3.1.1 Bosqua rathral denso
4.3. Feca recreo parrelaciones.
3122 Bosque trard framertato con pastos y outios
3.12 .1 Bosque Nutura Fragmentado con artustos
242 Mosico des pastiss y cultives
3.5 .1 Bospue plantido - conileras / eucal close
24.4 Mosaco de pastos y espacos nathunies

\begin{tabular}{|c|c|c|c|}
\hline$\Delta$ & $\begin{array}{l}\text { Barrera Orografica: } \\
\text { Restricción lispogifica } \\
\text { para la dispercion uttana }\end{array}$ & & $\begin{array}{l}\text { Area de dispersion urbana - suburbana } \\
\text { Asociacón de actividades nurales y } \\
\text { urbanas condominios, asentamientos } \\
\text { necorrales }\end{array}$ \\
\hline & Via - Aulopista & & Proyecto de tinel \\
\hline & Drensie-Cuenca & & Aeropuetto \\
\hline & $\begin{array}{l}\text { Centro poblacicral-municipal } \\
\text { infuenciado infuenciado por } \\
\text { dispersion urtana }\end{array}$ & & Embalses \\
\hline & $\begin{array}{l}\text { Perimetro } 20 \mathrm{Km} / \text { Cudad } \\
\text { intermedia. } \\
\text { Urbano - nural - franja fural urbana }\end{array}$ & A & Ecosistema Bosque Andino \\
\hline & Avea urtana consolidada & SA & Ecosistema Bcsque Stbandino \\
\hline & Corredores Ecoldogicos & BST & Ecosistema Bcsque Seco Tropical \\
\hline & $\begin{array}{l}\text { Remanentes Andinos en procesos } \\
\text { de conservacón }\end{array}$ & $\mathbf{P}$ & Ecosistema de Páramo \\
\hline
\end{tabular}
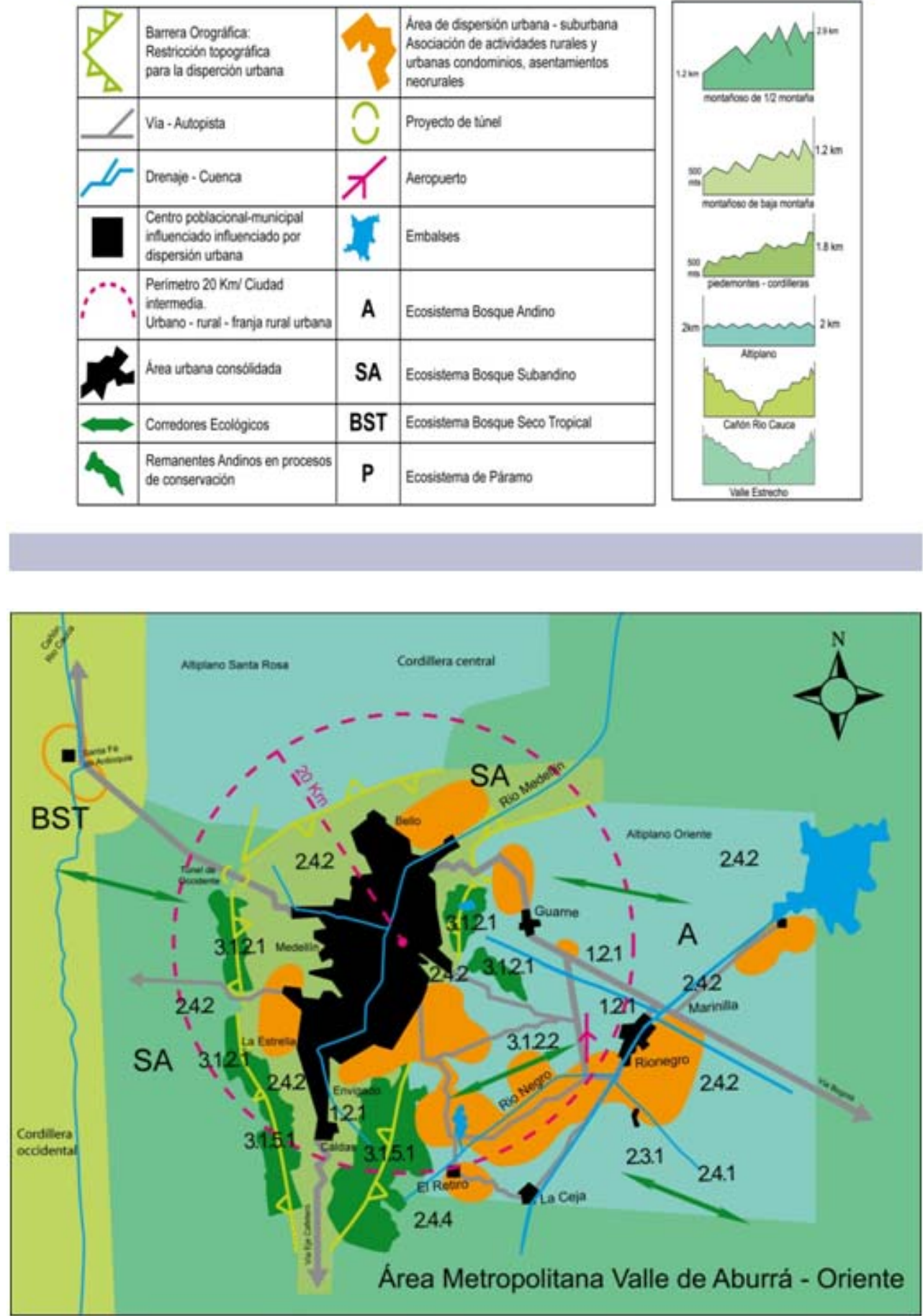
Figura 1. Organización espacial de la dispersion urbana entre el Area Metropolitana del Valle de Aburra y el altiplano del oriente antioqueño 2009 Fuente : elaboración del autor.

La incoherencia de la planificación conjunta ha llevado a que, en municipios como Rionegro, Medellín, Envigado y el Retiro, se afecte la dinámica ecológica de la cuenca alta del río Negro hacia sus áreas de nacimiento y en sus cubetas de inundación 0 humedales. Otro caso concreto se presenta en Manizales (figura 2), Pereira (figura 3) y Armenia (figura 4) donde la dispersión interfiere sobre los corredores que integran los ecosistemas andinos, sub andinos y secos tropicales. La autopista del café ha sido durante casi 10 años el eje de dispersión de estos asentamientos que vulneran el establecimiento de corredores establecidos entre el complejo nevado Ruiz Tolima y el valle del río Cauca en las cuencas de los ríos Chinchiná ${ }^{19}$, Otún, Consota, Barbas y Quindío.

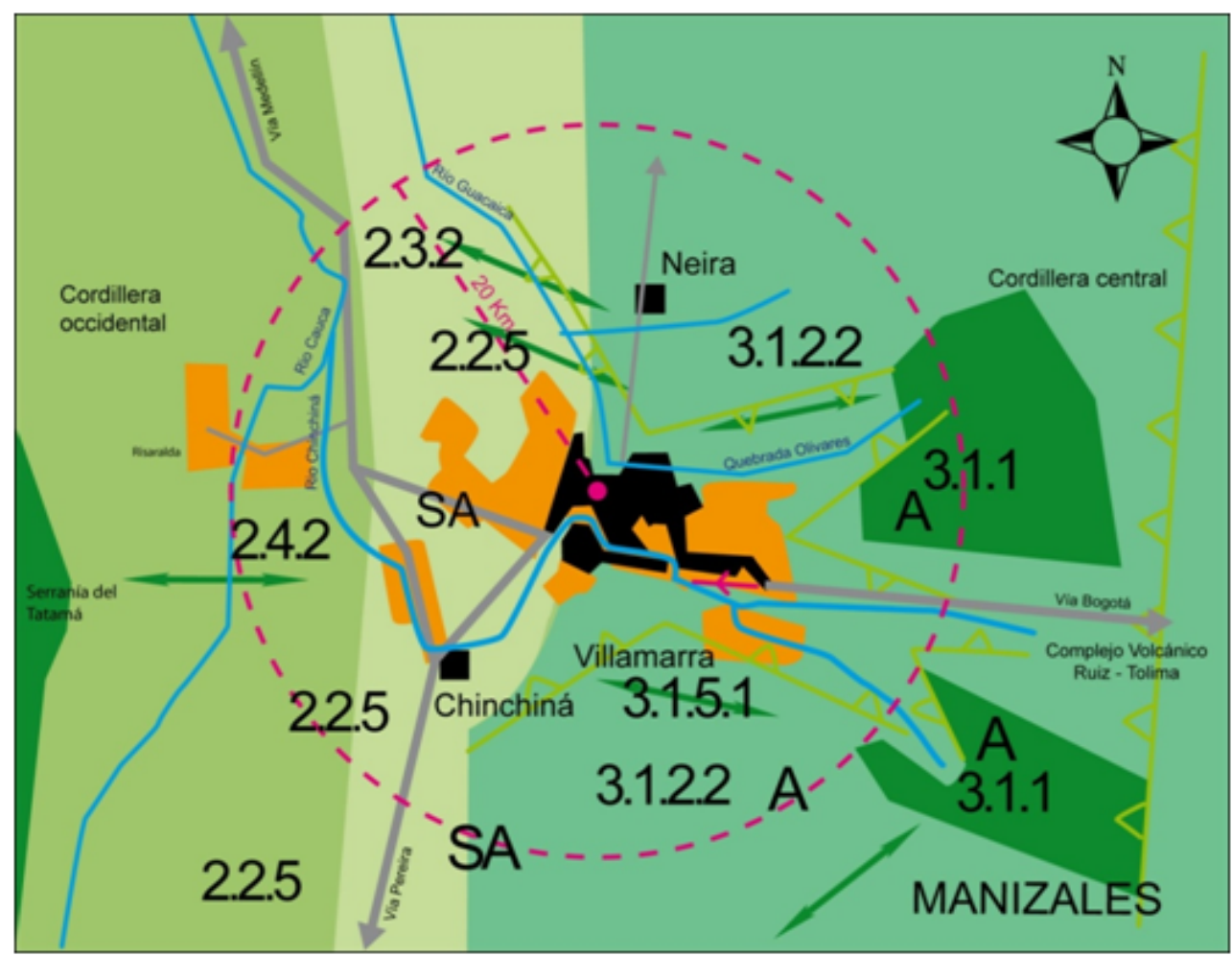

Figura 2. Organización espacial de la dispersion urbana del sistema urbano de Manizales 2009 Fuente : elaboración del autor. 


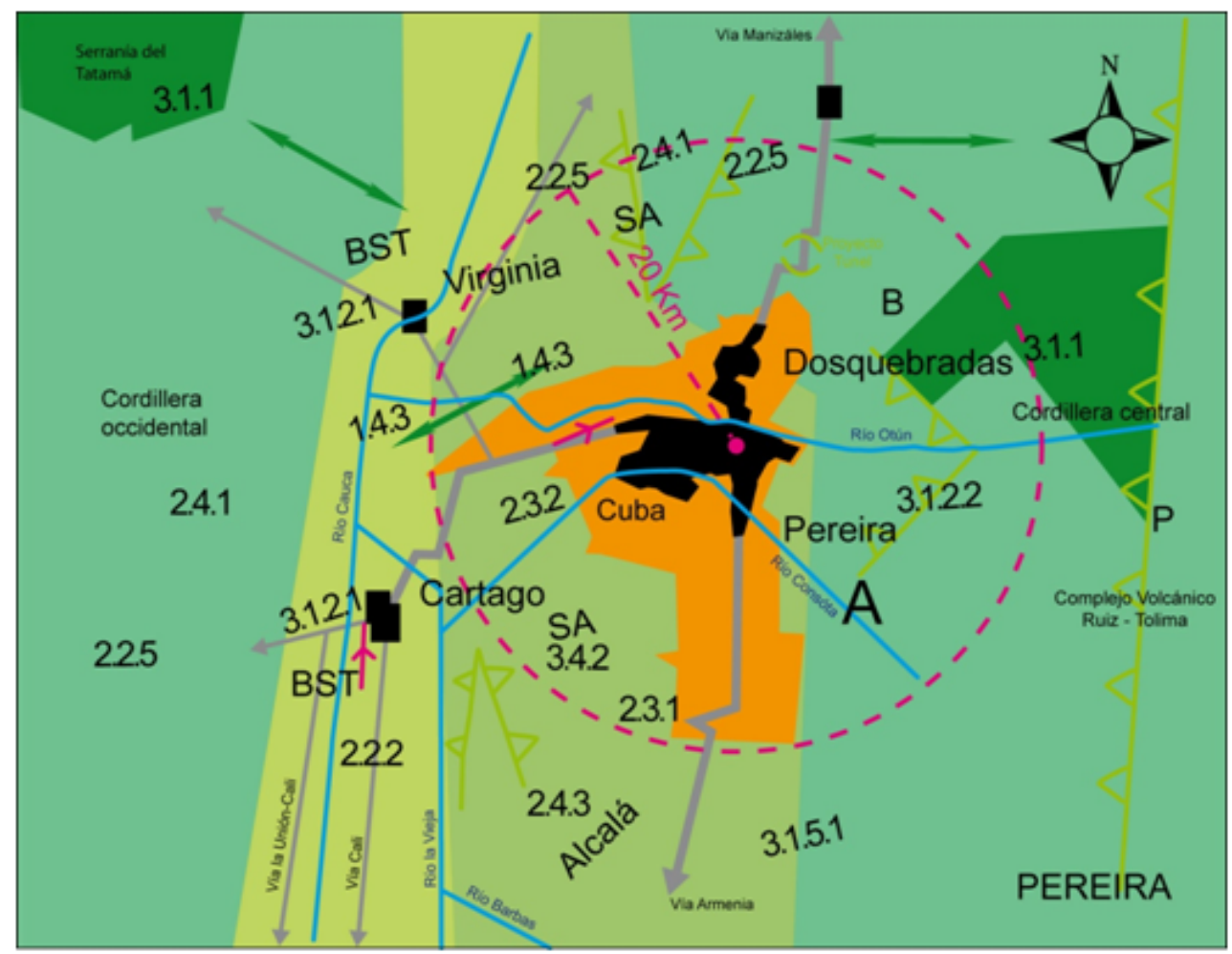

Figura 3. Organización espacial de la dispersion urbana del sistema urbano de Pereira 2009 Fuente : elaboración del autor.

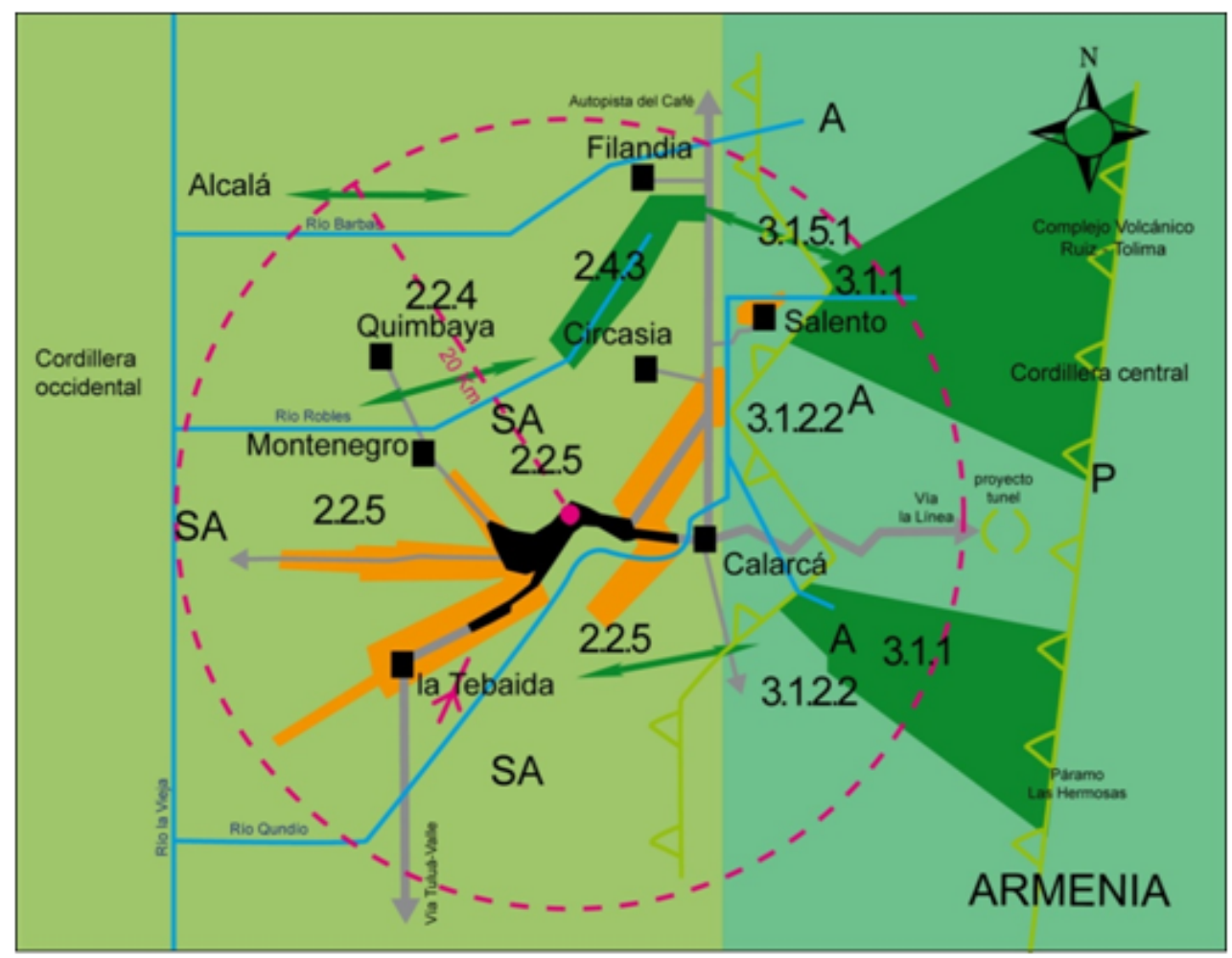

Figura 4. Organización espacial de la dispersion urbana del sistema urbano de Armenia, 2009 Fuente : elaboración del autor. 
Aunque son evidentes los esfuerzos realizados por parte de Corpocaldas, la CRQ del Quindío, CORPORISARALDA y la CVC del departamento del Valle, en sus trabajos de protección y promoción de identidades locales para la conservación de estos corredores, como en el caso de sus programas de conectores ecológicos rio Barbas- Bremen en los municipios de Cartago y Filandia, estos son susceptibles a las tendencias de parcelación dadas en sus proximidades.

Un hecho interesante a tener en cuenta para Antioquia y el eje cafetero es la relación costo beneficio de los bosques comerciales. Estos, por varias décadas, han funcionado como áreas de amortiguación entre ecosistemas fragmentados y la dispersión de asentamientos. En vista de que la permanencia de estos bosques depende de la rentabilidad del negocio comercial de la madera, no es posible garantizar su continuidad y, por ende, su función indirecta como conectores y amortiguadores. La deforestación realizada para beneficio maderero, permite en varios casos, después de realizada la tala, la parcelación inmediata de sus espacios, como se presenta en los municipios del Retiro y Envigado, y en el sur oriente de Pereira, donde en los últimos 8 años se ha extendido la venta de lotes para promover condominios.

En Salento, por ejemplo, municipio modelo a nivel Latinoamérica en procesos de conservación y participación, actualmente se presentan actividades turísticas y de urbanización proyectadas principalmente desde Armenia. De un tiempo atrás la parcelación sobre unidades agro-productivas al margen del río Quindío (sector de Boquía- Salento, San Juan de Carolina), ha incrementado la presión ecológica por contaminación y densificación poblacional. Las unidades eco-turísticas hacia el interior del valle del Cocora amenaza los estados de aislamiento de los bordes ecosistemas que interconectan páramos y selvas andinas-subandinas en la cuenca alta del río Quindío. En Manizales, a pesar de las restricciones topográficas que explican la compresión de su sistema urbano, la localización de unidades residenciales aisladas tiende a interferir sobre áreas de reservas declaradas en los planes de ordenamiento de Manizales y Villamaría.

En sí, el desarrollo espacial de los condominios plantea diferentes afectaciones en términos socioespaciales y ecológicos, aunque esto no es una generalidad ya que existen proyectos enmarcados sobre especificaciones correctas de urbanización en áreas de expansión e integrados a una planeación infraestructural. Pero, paralelo a esto, es evidente la proliferación de proyectos que, evadiendo figuras de reglamentación sobre la misma legalidad, logran posicionase en lugares no aptos para urbanizar.

La incidencia de los condominios sobre la estabilidad de las estructuras ecológicas y en las configuraciones sociales locales-regionales se podría por el momento definir en tres formas:

1. Las intervenciones físicas para la adecuación de condominios inciden en la estructura ecológica de los ecosistemas por medio de la remoción de los suelos vitales para regular su capacidad de reserva de humedad, así como el truncamiento de la red de drenaje y la obstrucción de posibles corredores.

Esta situación es evidente en el sector de Cerritos (vía Pereira, Cartago, la Virginia) donde los trabajos de excavación y explanación de terrenos comprometen la estabilidad hídrica y la conexión de corredores por el truncamiento de las quebradas ${ }^{20}$. Paralelo a esto, se presenta una deforestación al interior de los remanentes subandinos para dar espacio y encerramiento con el mismo bosque; aspecto que aprovechan los proyectos inmobiliarios para ofertarlo como "reservas ecológicas" propias del condominio

2. La proliferación de condominios descontextualizados de planes maestros de acueducto y alcantarillado, afectan las capacidades hidrogeológicas de las cuencas tras el incremento de los niveles de contaminación.

La ausencia de planes maestros de acueducto en los casos del triángulo el Retiro, Guarne, Rionegro en el oriente antioqueño, en Manizales hacia el río Cauca, en Villamaría en el sector de la Florida, en Cerritos en la confluencia entre Pereira, Cartago y la Virginia, pone al límite las capacidades de suministro de agua de los acueductos veredales y la capacidad de los remanentes forestales para proveer los sistemas de agua a los nuevos y antiguos sistemas de captación. Igualmente, la ausencia de un plan maestro de alcantarillado -no previsto en la expansión de condominios- ha obligado a que la mayoría de proyectos asuman la construcción de pozos sépticos ${ }^{21}$ aprobados casi indiscriminadamente por las Corporaciones Autónomas Regionales, sin un estudio previo sobre las afectaciones por las fugas de lixiviados por parte de éstos hacia la red hídrica superficial y subterránea. 
3. La parcelación consecuente a la deforestación de los bosques comerciales aproxima los condominios a remanentes en conservación e incrementa el efecto de borde sobre estos ${ }^{22}$.

En Manizales, por ejemplo, la dispersión de condominios hacia el oriente se aproxima cada vez más a las márgenes de las zonas de reservas hídricas y de biodiversidad andina de la reserva de Rioblanco, espacio que surte el acueducto de la ciudad. Caso similar sucede entre los municipios de Envigado y el Retiro, donde proyectos en curso, como el de Bosques de la Sierra, propone la urbanización de 480 hectáreas entre remanentes andinos y bosques comerciales declarados por los POT como áreas de protección $^{23}$

Estos tres aspectos demuestran que existe una responsabilidad en materia de cambio climático localregional por parte del desarrollo no planificado de las unidades residenciales. En el mismo orden, el hábitat del condominio no sería un hecho sin sus formas de consumo vitales que necesita, como por ejemplo la dependencia absoluta del vehículo y que, ante la proliferación espacial de este modo de vida, es evidente la saturación del tráfico vehicular; demandando en el tiempo ampliaciones infraestructurales y por lo tanto una mayor presión ecológica.

De esta manera, el condominio se puede considerar como un agente "colonizador urbano", sus externalidades conducen a afectaciones de predios rurales contiguos o al margen de las vías de acceso, desarrollando un ambiente propicio para atraer mercados inmobiliarios, desarrollo de sub centros de servicios y de comercio que promueven la competitividad regional y el desarrollo económico pero que, al mismo tiempo, genera un pasivo ambiental local-regional representado en la densificación poblacional próxima a las áreas de conservación y en el debilitamiento de las condiciones de sostenibilidad ecológico-ambiental.

En sí, la idea de un modelo urbano compacto es interesante frente a las posibilidades de interacción de los habitantes con entornos ecológicos controlados y segmentados, siempre y cuanto los proyectos garanticen las medidas de mitigación, corrección, compensación y prevención de esta antropización urbana en ecosistemas y unidades agrícolas. Pero, consecuente con la realidad, la iteración urbana descrita para los sistemas urbanos referidos repercute drásticamente sobre el conjunto ecológico funcional en cuencas hidrográficas del eje cafetero y el altiplano de oriente.

\section{COMENTARIOS FINALES}

En la actualidad, la red residencial dispersa ha elaborado un tejido que hace antesala al desarrollo espacial urbano para las siguientes décadas. A diferencia de las anteriores formas de crecimiento espacial urbano a lo largo de la segunda mitad del siglo XX, ésta presenta una dispersión más amplia gracias a las capacidades tecnológicas que la acompañan. La elaboración de este tejido está apolillando las estructuras y funciones vitales de los ecosistemas sobre las que se instala y vulnerando a su vez la oferta de servicios ambientales actuales y a futuro ${ }^{24}$.

Condominios, autopistas, zonas francas, industrias, centros comerciales, centros de servicios, clubes, programas de vivienda, asentamientos precarios, neorurales, universidades, hospitales, entre otros, son elementos y al mismo tiempo actividades que reconfiguran la espacialidad de la ruralidad circundante de las ciudades referidas en este trabajo. Vemos cómo sólo explorando las relaciones entre el condominio y la función de los ecosistemas andinos y subandinos, se encuentran una serie de alteraciones ecológicas locales, que de manera repetida por la dispersión en una escala regional, interrumpen el contexto general de los frágiles conectores entre ecosistemas y comprometen la calidad de las reservas de acuíferos ${ }^{25}$.

Este documento sólo presenta un esbozo comparativo de esta situación en las expansiones urbanas de algunas áreas antioqueñas y del eje cafetero, sin profundizar por el momento en la diversidad de transformaciones sociales que puede desencadenar la proliferación de los condominios. Éstas tendrían que ver con las dinámicas laborales, la modernización del campo, la segregación residencial, las formas de consumo, la privatización de los espacios, la seguridad privada y, tal vez lo más importante, la construcción de los conceptos de familia, ciudad y espacio urbano por parte de quienes habitan las periferias urbanas. 
Por el momento, es importante que las formas de ordenamiento desarrolladas por cada uno de los municipios involucrados en este fenómeno, superen sus diferencias y se integren comprometidamente en una planificación regional conjunta. En este aspecto existen avances significativos en materia de declaración de reservas ecológicas y en la proyección de planes maestros de acueducto y alcantarillado para el oriente antioqueño, el área metropolitana de Pereira y Armenia, con algunos de sus municipios próximos.

Por último, se espera que los propósitos actuales dados en el oriente antioqueño por medio del PLANEO, y los compromisos establecidos en la agenda integración urbano regional por parte de los comités técnicos de la eco región Eje cafetero, consideren los alcances y repercusiones ecológicas de la dispersión de los condominios y las regulación de las parcelaciones que permiten su promoción.

\section{BIBLIOGRAFÍA}

- $\quad$ Agenda para el desarrollo sostenible de la ecoregión eje cafetero- Colombia 2007-2019. (2007). Documento Técnico. AlmaMater, CVC, CORTOLIMA, CORPOCALDAS, CRQ, CARDER.

- AGUDELO, Luis. (2005). "La articulación urbano-regional: indicadores de sostenibilidad y ordenación del territorio. Huella ecológica y ecológica y ecosistemas estratégicos en Medellín." Región, Ciudad Y Áreas Protegidas. En: Colombia ISBN: 9588101212 ed: Cerec , v.1 , p.247 2661 ,

- $\quad$ ALLEN, Adriana. (2003). La interfase periurbana como escenario de cambio y acción hacia la sustentabilidad del territorio.cuadernos del CENDES Vol 20 ,No 53 http://www.scielo.org.ve/scielo.php?script=sci_arttext\&pid=S1012$25082003000200002 \&$ Ing=es\&nrm=iso>. ISSN 1012-2508.

- $\quad$ BARROS, Claudia. (1999). De rural a rururbano:" transformaciones territoriales y construcción de lugares al sudoeste del área metropolitana de Buenos Aires", en: Scripta Nova, Revista electrónica de Geografía y Ciencias Sociales, Universidad de Barcelona, n 45 (52), 1 de agosto 1999, Barcelona. http://www.ub.es/geocrit/sn-45-52.htm

- $\quad$ BETTINI, Virgilio. (1998). Ecología urbana, Barcelona.

- DÁVILA, Julio. 2003. Enfoques de intervención en la interfase periurbana.cuadernos CENDES, vol 20, No53, www.scielo.org.ve/scielo.php?script=sci_arttext\&pid=S101225082003000200003\&lng=en\&nrm=i so>. ISSN 1012-2508.

- $\quad$ DEMATTEIS, Giussepe. (1998). Suburbanización y periurbanizacion: ciudades anglosajonas y ciudades latinas, in: MONCLUS, Francisco. 1998. La ciudad dispersa, centro de cultura contemporánea, Barcelona.

- De MATTOS, Carlos (2001) Metropolización y suburbanización. En Rev. Revista Latinoamericana de Estudios Urbano Regionales, EURE, Santiago de Chile, Vol. XXVII, Núm. 80, Santiago: Instituto de Estudios Urbanos y Territoriales de la Pontificia Universidad Católica de Chile.

- $\quad$ DNP- DDUPA. (2009). Documento Conpes 3583. Lineamientos de política y consolidación de los instrumentos para la habilitación de suelo y generación de oferta de vivienda, Bogotá.

- $\quad$ FLÓREZ, Antonio. (,2003).Colombia: evolución de sus relieves y modelados, Universidad Nacional de Colombia, Bogotá,

- $\quad$ GÓmEZ, J, MUÑOZ JIMÉNEZ, J, ORTEGA, N. (1995). El pensamiento geográfico. Estudio interpretativo y antología de textos (De Humboldt a las tendencias radicales)Madrid, Alianza Textos

- $\quad$ HARVEY, David. (1985). Urbanismo y desigualdad social., Siglo XXI, Madrid, España.

- HERMELIN M, RENDON, D. (2007). "Medellín y el área metropolitana del Valle de Aburrá" Entorno natural de 17 ciudades de Colombia . En: Colombia ISBN: 978-958-8281-70-4 ed: FONDO EDITORIAL UNIVERSIDAD EAFIT , v.null , p.187 - 211 1,

HERMELIN M. (2008) "El hombre y su entorno natural en el Oriente antioqueño." Realidades y prospectiva del Valle de San Nicolás, Antioquia, Cámara de comercio del Oriente Antioqueño. En: Colombia ISBN: 978-958-98669-0-0

- LEFEBVRE, Henri (1979). De lo rural a lo urbano, Barcelona LÓPEZ, Fabiola. (2004). Estructura y función e Bordes de Bosque. Ecosistemas, enero-abril. Año/vol. XIII,numero 001, Asociación Española de Ecología Terrestre, alicante España http://148.215.1.166:89/redalyc/pdf/540/54013110.pdf 
RODRÍGUEZ, N, ARMENTERAS, D, MORALES,M, ROMERO,M. (2004). Ecosistemas de los andes colombianos. Instituto de investigación de recursos Biológicos Alexander Von Humboldt, Bogotá, Colombia

- SutTON, David. (2003). Fundamentos de ecología. Limusa, México Plan de gestión ambiental regional- PGAR 2003-2012. (2002). Gobernación del Quindío.http://www.cortolima.gov.co/2006/index.php?option=com_content\&task=view\&id=261\&lt emid $=447$

TACOLI, Cecilia. (1998). Rural-urban interactions: a guide to the literature, in: Enviromental and urbanization 10(1) págs 147-66

- VAN DER HAMMEN, T, DOS SANTOS, A. (1995). Estudios de ecosistemas tropoandinos. La cordillera central colombiana transecto Parque de los Nevados. J. CRAMER, Berlín, Stuttgart.

1. Geógrafo, MSc Hábitat Universidad Nacional de Colombia, docente investigador del Instituto de Estudios Regionales INER, docente de la Maestría en Estudios Socioespaciales, grupo de Estudios del Territorio- GET, Universidad de Antioquia, coordinador de extensión del INER. El investigador se ha desempeñado como docente en Geografía Urbana y Biogeografía en la Universidad de Caldas e investigador en el campo de la gestión de riesgos con el IDEA, sede Manizales. 2. No existen cifras concretas que midan la espacialidad de la dispersión urbana y no se podría referir esto sólo con los datos de tasas de crecimiento poblacional y de licencias de construcción en cada unos de estos sistemas. La dispersión urbana agrega con el tiempo espacios político administrativos correspondientes a los municipios que gravitan en torno a Bogotá y el área metropolitana de Aburrá. En tal sentido, es factible el hecho de las prolongaciones urbanas que se impulsan desde el área metropolitana hacia el altiplano de oriente y que se conjugan con el crecimiento urbano proyectado principalmente desde el Municipio de Rionegro. Igual es el caso del distrito capital, su dispersión urbana se amplifica sobre el altiplano de la sabana de Bogotá y de igual forma se entreteje con las tendencias de crecimiento urbano proyectadas desde Fontibón, Chía y Zipaqirá desarrollando un mosaico de actividades industriales ligadas a las zonas francas y programas de vivienda que reducen las áreas de producción agrícola establecidas en la cuenca media del Rio Bogotá 3. Formulación y acompañamiento a las mesas técnicas del Hábitat en la eco región Eje cafetero, abril 2005. La Tebaida, Quindío, Universidad de Caldas, Alma Mater, Corporación Autónoma del Quindío, Universidad Autónoma de Manizales, Universidad Gran Colombia. Quindío. 4. Participación en el Plan estratégico Habitacional Medellín 2022 julio de 2009, Foro Territorio Sin Fronteras, agosto de 2009, Foro ambiental Regional, Medellín abril de 2009. 5. Referido a tejidos urbanos capilares de cordones o vías que interconectan globos que en este caso son parcelas urbanizadas 6. Excepcionalmente el proyecto en curso por parte de Arquitectura y Concreto, llamado Sierra grande en el Municipio del Retiro; comprende 481 hectáreas, es decir , 4.810 .000 millones de metros cuadrados para construir 481 viviendas y de las cuales 270 serían inmuebles de tres niveles. El colombiano, 30 de septiembre de 2008. Esto hace suponer el diseño de lotes de 10.000 metros cuadrados, un consumo de suelo exagerado si se mira desde la equidad social del espacio ante los discursos de escases de suelo urbano en las ciudades colombianas. 7. En la actualidad, los costos aproximados de metro cuadrado en diferentes áreas de proyectos de condominio son: Medellín vía las Palmas, Loma del Escobero 50 - 115 \$US, el Retiro, Rionegro- sector de Llanogrande 25 - 60 \$US, Manizales, 25 - 40 \$US, Pereira, Cerritos, la Virginia 15- 60 \$US, Armenia, La Tebaida, Calarcá 25- 50\$US. Datos suministrados por http://colombia.inmobiliaria.com/, revistas de informe inmobiliario Antioquia, Construoferta, Caldas, Risaralda, años 2008-2009. 8. Relación de agotamiento de los suelos aptos para urbanizar en los sistemas urbanos, considerando su necesidad de ampliarse espacialmente. Igualmente el concepto trata sobre la escasez de recursos para adelantar las acciones de gestión y financiación que permitan la habilitación de suelo. Esto afecta el desarrollo, la viabilidad financiera de programas de vivienda particular y, especialmente, a la vivienda de interés social. Para profundizar más en esto el documento COMPES 3583 DE 2009, expone una marcada disminución de los suelos aptos para urbanizar las ciudades colombianas. 9. Referidos a unidades de relieve o modelado reconocidos en escalas cartográficas medias 1:200.0001:100.000, Flórez

(2005:257). 10. El estudio de ECOSISTEMAS TROPO ANDINOS de 1995 adoptó este concepto para definir el contexto espacial del eje volcánico y de sistemas glaciares residuales con altitudes entre 3.000 y 5200 m.s.n.m localizado en los departamentos de Caldas, Tolima, Risaralda y Quindío. Esto integra en su contexto un sistema de páramos en los que se originan las cuencas hidrográficas de los ríos Chinchiná, rio Claro, Otún y Quindío hacia la vertiente occidental de los andes centrales colombianos. 11. Fragmentos espaciales de un ecosistema preexistente por actividades antrópicas, situación que 
limita las capacidades funcionales y estabilidad del mismo. Una solución para garantizar su estabilidad tiene que ver con el diseño de corredores ecológicos que puedan conectar la mayor cantidad de fragmentos. En diferentes planes de desarrollo municipales de los municipios del oriente antioqueño, está contemplado el diseño de varios corredores orientados por el curso del río Negro y sus afluentes, con el fin de preservar las relaciones ecológicas del interior del altiplano. Corpoceam y Cornare son instituciones que durante más de 20 años vienen liderando estas propuestas para el caso del oriente. Igualmente Corpocaldas, Corporirsalralda, la CRQ del Quindío y la CVC del valle, junto a los comités departamentales cafeteros, han trabajado en el desarrollo de un plan de gestión ambiental regional PGAR 2003-2012 que promueve estrategias de conservación, considerando la promoción de corredores como una alternativa.

12. Se establece en altitudes entre 2350 y 3750 m.s.n.m, se registra la presencia de 191 especies de aves. La selva andina se caracteriza por sus condiciones de niebla y nubosidad permanente, aspecto determinante en el crecimiento de la flora, por lo cual desarrollan adaptaciones especiales en el tamaño de sus hojas. La estratificación de la selva andina no supera los 20 metros de altitud y con presencia de helechos arbóreos y palmas. 13. Se enmarca en la zona caficultora de los andes entre los 1.500 y 2.000 m.s.n.m y corresponden a bosques húmedos con presencia de robledales con gran amplitud altitudinal de 20 a35 metros de altura, con estratos emergentes discontinuos de dos a tres estratos. Se caracteriza por la abundancia de palmas, abundancia de epífitas, orquídeas y helechos arbóreos y un número apreciable de elementos australes sudamericanos. Se han reconocido aproximadamente 162 especies de aves. 14. El bosque seco tropical es un enclave de biodiversidades adaptada en los espacios donde los niveles de humedad son bajos (precipitaciones inferiores a los 1200- $1500 \mathrm{~mm}$ ). Su importancia en un contexto local y regional tiene que ver con la diversidad de insectos y aves que habitan allí, y su función como agentes polinizadores en este ecosistema y el subandino. En Colombia, el bosque seco tropical se localiza en los piedemontes de cañones y valles de las depresiones estructurales del Magdalena y Cauca, en depresiones estructurales internas de las cordilleras como en el caso de la depresión del Patía en Nariño y la depresión del cañón del rio Chicamocha en Boyacá- Santander. 15. En el trabajo de Sutton sobre fundamentos de ecología (2003), se considera que todos los ecosistemas son sistemas abiertos debido al intercambio de energía y la producción de energía interna y con el exterior. Estos procesos dependen de los ciclos bio geoquímicos del agua, radiación que produce energía o alimento al tiempo que se presenta un flujo de elementos genéticos de microorganismos. Por lo tanto, el funcionamiento de éste depende de la formación de estratificaciones, comunidades bióticas, y sucesiones ecológicas que garantizan la estabilidad del mismo en su distribución de la temperatura, los niveles de humedad en retención y transporte, los grados de descomposición y producción de orgánica. 16. De forma natural el corredor ecológico se dispone orientado por las márgenes de los drenajes, estos tienden a conservarse de manera más prolongada cuando permanecen en relieves montañosos abruptos, como en el caso de Manizales y Villamaría, Armenia y Calarcá, donde los programas de productividad cafetera y forestal comercial integran estos corredores a propósitos de conservación y diseño de reservas hídricas en torno a los acueductos. En el caso de los corredores existentes entre Pereira y Armenia, la morfología del relieve de abanicos permite una mayor presión de las actividades humanas hacia los corredores establecidos en los ríos Otún, Consota, Barbas y Quindío. La autopista del café corta estos corredores a la vez que imprime un cambio en la vocación de usos. A pesar de esto, son de resaltar los trabajos de reforestación con especies nativas sub andinas que funcionan como corredores e integran remanentes entre los ríos Barbas y Bremen en el municipio de Filandia. Esta ha sido una labor piloto en la región que ha sido liderada por parte de la CRQ y el Instituto de Investigaciones para la Biodiversidad Alexander Von Humboldt, en el cual se ha podido, en una década de trabajos entre propietarios ganaderos y de bosques comerciales y la comunidad, el establecimiento de programas de conocimiento, conservación y utilización de estos corredores. 17. La agenda para el desarrollo de la eco región Eje cafetero 2007-2009 ha establecido como meta el potenciamiento de actividades productivas sostenibles aprovechando la plataforma de los recursos naturales de agua, suelos y los sectores sociales culturales que lo conforman. Contrariamente a lo expuesto, los desarrollos infraestructurales y la promoción de cadenas productivas y cluster de mercados eco turísticos, ganaderos y de bosques comerciales ejercen una presión negativa en la sostenibilidad ecológica de los suelos por medio del cambio de vocación de éstos (transformación del paisaje cultural cafetero por escenarios inmobiliarios residenciales, turísticos, agroindustriales) y de la biodiversidad, situaciones concretas dadas a lo largo de la autopista del café. 18. El apolillamiento es una acción de roer, penetrar o destruir un tejido por parte de un agente. La interpretación de imágenes de satélite provistas por Google Earth 2007para el oriente antioqueño, permite identificar salpullidos de inserciones espaciales urbanas sobre estructura ecológico espaciales (cuenca de río Negro) que afectan dinámicas de calidad, cantidad del agua y permanencia de ecosistemas. Principalmente, se reconoce la inserción dispersa de unidades residenciales y que definen la escaza planeación espacial de las parcelaciones. 19. El proyecto de autopista del café entre Manizales vía Medellín y vía Chinchiná, interrumpió la 
continuidad del mosaico cafetero en el cual existía una asociación entre fragmentos de bosques y cafetales. El trazado se convirtió, con el tiempo, en una barrera para la migración de pequeños mamíferos y anfibios que hacen parte del ecosistema subandino. 20. Particularmente donde existen inclinaciones empinadas en relieves montañosos como en Manizales, Envigado y El Retiro, las obras de adecuación de terrenos aceleran la erosión y sedimentación, afectando la calidad del agua superficial y subterránea. El uso de maquinaria pesada y el acopio de materiales, compactan el suelo, haciéndolo menos permeable, destruyendo su estructura y deformando los niveles freáticos locales. Esto conduce al desarrollo de erosiones superficiales y profundas manifiestas en el hundimiento, los deslaves, y demás fallas mecánicas del suelo y subsuelo. El apolillamiento de los condominios en los casos de Cerritos-Pereira y en el sector de Llanogrande en Rionegro hace que disminuya la cantidad de agua subterránea a razón de proliferación puntual de áreas impermeables (pavimentaciones), además de la alteración de los patrones naturales de drenaje. Aumenta el escurrimiento, resultando en la erosión y sedimentación. 21. El tanque o pozo séptico es un sistema de tratamiento de aguas residuales domésticas que consiste, en términos generales, de una cámara cerrada a donde llegan los residuos, la cual facilita la descomposición y la separación de la materia orgánica por acción de las bacterias de estos residuos. El nitrato es uno de los contaminantes inorgánicos que genera mayor preocupación en la contaminación de aguas subterráneas y que proviene de lagunas de retención de desperdicios sólidos no cubiertas por debajo, la infiltración de aguas residuales no tratadas y pozos sépticos que no estén funcionando bien. http://www.atsdr.cdc.gov/es/general/agua/es_groundwater4.html

22. López (2004) define el efecto de borde como un concepto el cual comprende un amplio espectro de procesos, influencias mutuas y flujos ecológicos que pueden resultar en cambios en la estructura y composición de los bordes y hábitats adyacentes. Una dispersión de estos asentamientos en kilómetros afectan las cercanías inmediatas de los bosques por medio del flujo vehicular y el incremento del ruido. Esto altera el comportamiento de la movilidad de los individuos. Tanto actividades rurales como urbanas reducen la dispersión de semillas y la posibilidad de la depredación de nidos de aves en los bordes ante una ausencia de revegetalización y desarrollos arbustales en los potreros para el caso rural. Pero igualmente, habría que tener en cuenta los factores como la edad, orientación e historia del uso del borde y hábitat adyacente anteriores a la instalación del condominio, para así conocer con certeza el impacto. El proceso de deforestación en la adecuación de estos asentamientos conduce a cambios en los patrones de la vegetación y micro climáticas (humedad del suelo, radiación, temperatura) entre el borde $y$ el centro del bosque. 23. En la actualidad CORNARE afirma que esta obre técnicamente no ha cumplido los requisitos porque no tiene la calidad de protección ambiental ya que parte de la obra se localiza sobre zonas de protección, es decir, áreas de amortiguamiento y bosques andinos. A pesar de esto, las obras de excavación, rellenos y nivelaciones del terreno continúan, poniendo en cuestionamiento la manera cómo el municipio del Retiro está otorgando las licencias ambientales de las parcelaciones. El colombiano, 24 de julio de 2009 24. El considerar los corredores ecológicos como canales de transferencia de biodiversidad y los bordes de los fragmentos boscosos como espacios del intercambio de materia y organismos entre dos hábitats, tiene importantes implicaciones en términos del conocimiento, uso y conservación, así como en el manejo de la vida silvestre en el contexto de la ecología urbana. 25. Los nitratos generalmente se mueven lentamente en el suelo y las aguas subterráneas: existe un lapso de tiempo de aproximadamente 20 años entre la actividad de contaminación y la detención de contaminantes en el subsuelo y aguas subterráneas. Por esta razón, se predice que las actividades actuales de contaminación continuaran afectando a niveles de nitrato por varias décadas. http://www.lenntech.es/agua-subterranea/nitratos.htm\#ixzz0RBtRC9Yv 